\title{
Single-pass high-gain tapered free-electron laser with transverse diffraction in the postsaturation regime
}

\author{
Cheng-Ying Tsai, Juhao Wu, ${ }^{\dagger}$ Chuan Yang, ${ }^{\ddagger}$ Moohyun Yoon, ${ }^{\S}$ and Guanqun Zhoull \\ SLAC National Accelerator Laboratory, Menlo Park, California 94025, USA
}

(Received 29 January 2018; published 28 June 2018)

\begin{abstract}
It has been well known that the resonant interaction of an ultrarelativistic electron beam and the radiation field in the single-pass high-gain free electron laser (FEL) amplifier leads to the optical gain guiding. The transverse Laplacian term of the slowly varying wave equation in the linear regime can be approximated as a constant detuning parameter, i.e., $\left|\nabla_{\perp}^{2}\right| \sim k_{R} / z_{R}$ where $k_{R}$ is the resonant wave number and $z_{R}$ is the Rayleigh range of the laser. In the post-saturation regime, the radiation power begins to oscillate about an equilibrium for the untapered case while continues to grow by undulator tapering. Moreover, in this regime the gain guiding decreases and the simple constant detune is no longer valid. In this paper we study the single-pass high-gain FEL performance in the post-saturation regime with inclusion of diffraction effect and undulator tapering. Our analysis relies upon two constants of motion, one from the energy conservation and the other from the adiabatic invariant of the action variable. By constructing a two-dimensional axisymmetric wave equation and the coupled one-dimensional electron dynamical equations, the performance of a tapered FEL in the postsaturation regime can be analyzed, including the fundamental mode profile, the power efficiency and the scaled energy spread. We begin the analytical investigation with two different axisymmetric electron beam profiles, the uniform and bounded parabolic ones. It is found that the tapered FEL power efficiency can be smaller but close to the taper ratio provided the resonant phase remains constant and the beam-wave is properly matched. Such a tapered efficiency is nearly independent of transverse electron beam size before significant electron detrapping occurs. This is essentially different from the untapered case, where the power extraction efficiency is around the essential FEL gain bandwidth (or $\rho$, the Pierce or FEL parameter) and depends on the beam size. It is also found that the power enhancement due to undulator tapering is attributed more by the field increase outside the transverse electron beam than that inside the transverse electron beam. Several scaling properties on the taper ratio and the transverse electron beam size are also discussed in this paper.
\end{abstract}

DOI: 10.1103/PhysRevAccelBeams.21.060702

\section{INTRODUCTION}

Generating an intense high-power x-ray free electron laser (FEL) can be of great interest, e.g., the peak power at the level of terawatt (TW) or sub-TW, since such power level of

\footnotetext{
jcytsai@SLAC.Stanford.EDU

jhwu@SLAC.Stanford.EDU

"Visiting Ph.D. student from National Synchrotron Radiation Laboratory, University of Sciences and Technology of China, No. 42 Hezuohua South Road, Hefei, Anhui 230029, China.

${ }^{\S}$ On sabbatical leave from Department of Physics, Pohang University of Science and Technology, Pohang 37673, Korea.

Visiting Ph.D. student from Institute of High Energy Physics, Chinese Academy of Sciences, and University of Chinese Academy of Sciences, 19B Yuquan Road, Shijingshan District, Beijing 100049, China.
}

Published by the American Physical Society under the terms of the Creative Commons Attribution 4.0 International license. Further distribution of this work must maintain attribution to the author(s) and the published article's title, journal citation, and DOI. output radiation has stimulated numerous experiments in various scientific areas (see, for example, Refs. [1-3]). For example, in an x-ray nanocrystallography study [1] a laser beam of 1.8-keV photon energy and $~ 100-$ femtosecond (fs) pulse duration, with about $10^{12}$ photons per pulse on the sample was experimented. Research in these areas will greatly benefit from at least 10 to 100 times larger number of coherent photons within a comparable or shorter pulse duration than the typical existing x-ray FELs [4-7]. That is, the requirements will correspond to an output laser consisting of $10^{13}$ to $10^{14} 1-\mathrm{keV}$ photons within a several tens of fs pulse duration, i.e., the target output power of $1 \mathrm{TW}$ or higher.

The output characteristics of FEL are determined by its operation modes. In the x-ray wavelength regime a singlepass high-gain FEL can work either in the self-amplified spontaneous emission (SASE) or seeded mode, despite the lack of direct seeding source. In the SASE mode [8], the initial seeding originates from shot noise of the electron beam. Therefore the output characteristics of SASE FEL 
can be noisy in both temporal and spectral profile, although the transverse coherence can be excellent see, for example, Refs. [9-11] and references therein. Acting as an amplifier, the seeded mode indeed requires an input source. It has been known that utilizing higher harmonics generation, e.g., high-gain harmonics generation (HGHG) $[12,13]$ or echo-enabled harmonic generation (EEHG) $[14,15]$ can be an option. Another option is the so-called self-seeding [16-18]. In the self-seeding option the FEL system starts with the first section of undulators based on SASE mode and is followed by a crystal monochromator or gratings to purify the output spectrum, serving as the subsequent input signal. Then a second section of undulators proceeds and amplifies the (purified) signal up to saturation. Compared with SASE, the output characteristics of seeded FEL are in general with much narrower spectral bandwidth and better wavelength stability (see, for example, Refs. [16-19]).

Up to the end of exponential growth in the shortwavelength single-pass FEL, the power efficiency (defined as the ratio of the FEL radiation power to the electron beam power) is about $\rho$ (the Pierce or FEL parameter, usually smaller than $1 \times 10^{-3}$ for $x$-ray FELs), indicating that the output peak power can be $P_{\text {rad }} \approx \rho P_{\text {beam }}$ (with $P_{\text {beam }}$ the electron beam power) $\sim 25 \mathrm{GW}$ for an electron beam with a typical peak current $\sim 5 \mathrm{kA}$ and the nominal energy of several $\mathrm{GeV}$ in a 100 -m-long untapered undulator. There is still a factor of 20-40 before reaching the aforementioned sub-TW or TW power level. Dedicated undulator taperings are a typical technique for power enhancement [20] and recently the efficiency enhancement based on a phase jump method is also proposed [21,22]. Both techniques will favor seeded or self-seeded FELs. There have been numerous numerical simulations and multi-dimensional optimization schemes reported for highest achievable power levels [23-25].

The resonant interaction of ultrarelativistic electron beam and radiation field in the single-pass high-gain exponential regime of the FEL amplifier has been intensively studied, for example, the longitudinal microbunching dynamics associated with the exponential growth of the dominant modes and the SASE FEL statistical properties (see, for example, Refs. [9-11]). In the transverse dimension the well-known optical gain guiding, the compensation effect between the natural tendency of radiation diffraction and the presence of cooperating electron beam, was first predicted in 1980s [20,26,27] and later further completed in more dedicated theories, numerical simulation and experiments [28-33]. The transverse Laplacian term in the slowly varying wave equation in the exponential-growth regime can be well approximated as a constant detuning parameter, i.e., $\left|\nabla_{\perp}^{2}\right| \sim k_{R} / z_{R}$ where $k_{R}$ is the resonant wave number and $z_{R}$ is the corresponding Rayleigh range of the laser. This diffractive effect becomes more important for singlepass FELs at shorter wavelengths with ever-increasing undulator lengths, because the corresponding Rayleigh length can usually be only about 10-20 $\mathrm{m}$ and the shortwavelength radiation beam is difficult to confine by a waveguide structure. Here we remind that the Rayleigh range $z_{R}=\pi w_{0}^{2} / \lambda_{R}$ is inversely proportional to the radiation wavelength $\lambda_{R}$ while the diffraction effect itself $\left|\nabla_{\perp}^{2}\right|$ is wavelength independent and the key parameter is the waist of the radiation $w_{0}$. In this regime the transverse diffraction effect approximated as a constant detuning is no longer valid and further analysis is needed.

In the saturation regime the analysis of FEL dynamics becomes more involved. Employing the typical collective variables [34] to analyze the system dynamics is insufficient. This is primarily because the electron beam phase space distribution is not only characteristic of linear density and energy modulations but also begins to rotate, i.e., the electron beam executes the synchrotron motion. Such a synchrotron oscillation is due to the electron trapped in the ponderomotive potential well, formed by external undulator magnetic field and the appreciable saturated radiation field (the main signal). The resonant interaction between the main signal and the trapped electrons, once forming a positive feedback, will drive the electron synchrotron oscillation and feature a growing sideband signal in the FEL output spectrum, leading to the sideband instability [20]. This longitudinal instability mechanism has been known as a showstopper for reaching even higher FEL peak power in the post-saturation regime [35-40]. Moreover, there is another mechanism in the transverse domain, i.e., the focus of this paper, due to reduction of the refractive gain guiding in the post-saturation regime. This is relevant to reaching higher peak powers in FELs. The reduction of gain guiding is attributed by the less rapidly growing main signal and gradually increasing radiation beam size. Such a phenomenon has been observed and studied in various numerical simulations (see for example Ref. [23]). It deserves here to mention a recent work by Schneidmiller et al., [41] that they performed a comprehensive study of optimization of the short-wavelength FEL amplifier performance with emphasis on the dependence of the radiation diffraction effect. However studies based on analytical analyses are still constrained and were only limited for the constant-parameter (untapered) case [42,43]. A similar study is found in Refs. [44,45], where they treated the problem by using Green function method but assumed the explicit separation of the longitudinal and transverse radial dependence of the beam. Our presented analytical analysis enables to find some useful scaling properties, including the taper ratios and the transverse beam sizes. This serves as a motivation of this work.

In this paper we carry forward the previous work by Gluckstern et al. [42] on the studies of untapered FEL saturation dynamics, where they used the two invariants of motion, one from the energy conservation and the other from the fact of $z$-independent system Hamiltonian (where $z$ is the longitudinal undulator coordinate). We extend the 
analysis to the tapered case. In the presence of undulator tapering the Hamiltonian depends on $z$ and is no longer a constant of motion. However, if the fractional change of the undulator tapering strength is slow compared with the synchrotron period, the corresponding action variable can be still considered as a constant, i.e., the adiabatic invariant. Furthermore, according to the conclusion of Ref. [42], the equilibrium state of trapped electron phase space distribution at the initial saturation appears to most resemble the Boltzmann distribution. Using the two pieces of information we can derive some electron beam properties at the initial saturation. We note that the validity of the above analytical operation relies on the fact that the background of untrapped electrons is nearly independent of the system Hamiltonian [42]. Further analysis in the situation of appreciable electron detrapping has however been beyond the scope of this paper. The above discussion refers to the electron dynamics. For radiation field evolution we employ the two-dimensional axisymmetric wave equation to study the transverse dependence of the radiation beam profile with diffraction and in the presence of cooperating electron beam. In the analysis we consider two transverse electron beam profiles allowing analytical solutions: the uniform and the parabolic ones, and finally numerically compare with a case done by three-dimensional (3-D) simulations. We comment that although more practical issues emphasize how to extract more FEL power or energy from the electron beam, the presented results here can give us more understanding and provide further insights of the post-saturated tapered FEL performance.

This paper is organized as follows. In Sec. II A we briefly review the one-dimensional (1-D) FEL dynamics and the associated two constants of motion that will be employed in our subsequent analysis. In the same subsection we also discuss a simple scaling property of the FEL power performance with respect to the intrinsic FEL or Pierce parameter $\rho$ and to the ratio of undulator tapering. The equilibrium solutions, referred to as the starting location of undulator tapering, for the electron beam and radiation beam are then discussed and employed to derive several electron beam properties for later use in Sec. II B. After the preparations, we extend the 1-D wave equation to twodimensional (2-D) axisymmetric one with inclusion of transverse Laplacian operator to account for diffraction effect, and solve the resultant radiation field profile in the presence of driving electron bunching. The results are derived for two transverse electron beam profiles that allow analytical solutions. In Sec. III A we discuss the scaling properties of the above derived results to the transverse electron beam size and to the different levels of undulator tapering, including the special case of an untapered FEL. In Sec. III B we illustrate the spatial evolution of the radiation field intensity along the undulator coordinate $z$ and the transverse dimension for untapered and $8 \%$ tapered cases. The obtained results are consistent with the conclusion made in our analysis. Finally we summarize the results and discuss possible future work in Sec. IV.

\section{THEORETICAL FORMULATION}

In this section we formulate the problem by starting the investigation of 1-D high-gain FEL with a tapered helical undulator and later extending to 2-D for the radiation beam. Our primary focus will be in the post-saturation regime. The FEL process initiates from the following resonance condition

$$
\frac{\lambda_{R}}{\lambda_{u}}=\frac{1+K_{0}^{2} / 2}{2 \gamma_{R 0}^{2}},
$$

where $\lambda_{R}$ is the radiation wavelength of the main signal, $\lambda_{u}$ is the undulator period, $\gamma_{R 0}$ is the initial electron reference energy in units of its rest mass energy, $K_{0}$ is the (peak) dimensionless undulator parameter. In the presence of undulator tapering, the undulator parameter is in general a function of the undulator axis $z$, i.e., $K_{0} \rightarrow K(z)$. Here we consider $K$-tapering and assume $\lambda_{u}$ is a constant. The variation of $K(z)$ should follow with that of electron energy loss and ensure that $\lambda_{R}$ remains constant. For simplicity we do not consider the postsaturation dynamics of harmonic lasing FEL (see for example Ref. [46-48] and references therein).

\section{A. Model equations for tapered FEL saturation}

In the following we begin by defining the undulator taper profile $f_{B}(z)$ such that $K(z)=f_{B}(z) K_{0}$, where $0 \leq f_{B}(z) \leq 1$. For convenience we also define $\Delta \equiv$ $1-f_{B}\left(z_{f}\right)$ as the total taper ratio at the undulator exit $z_{f}$. The 1-D FEL process can be formulated based on the following single-particle Hamiltonian,

$\mathcal{H}\left(\theta_{j}, \eta_{j} ; \hat{z}\right)=\frac{\left(\eta_{j}-\eta_{R}\right)^{2}}{2 f_{R}}-i \frac{f_{B}(\hat{z})}{f_{R}(\hat{z})}\left(\mathcal{E} e^{i \theta_{j}}-\mathcal{E}^{*} e^{-i \theta_{j}}\right)$,

where $j\left(=1,2,3, \ldots, N_{e}\right)$ is the index for each individual particle and $N_{e}$ is the total number of macroparticles (electrons). $\theta=\left(k_{R}+k_{u}\right) z-\omega_{R} t$ is the electron phase with respect to the radiation, $k_{R}=2 \pi / \lambda_{R}$, and $\omega_{R}=c k_{R}$, $\eta \equiv\left[\gamma-\gamma_{R}(0)\right] / \rho \gamma_{R}(0)$ is the normalized energy deviation with respect to the dimensionless FEL or Pierce parameter (in CGS units):

$$
\rho=\frac{1}{\gamma_{R}(0)}\left(\frac{4 \pi e^{2} n_{0} K_{0}^{2}}{32 m_{0} c^{2} k_{u}^{2}}\right)^{1 / 3},
$$

with $e$ the charge unit, $n_{0}$ the volume density of the electron beam, $m_{0}$ is the electron rest mass. $\eta_{R}=\left[\gamma_{R}-\gamma_{R}(0)\right] / \rho \gamma_{R}(0)$. For convenience of subsequent analysis we introduce the normalized longitudinal coordinate $\hat{z}=2 k_{u} \rho z$, with $k_{u}=2 \pi / \lambda_{u}$. In the case of undulator tapering, the electron 
reference energy is modified accordingly through the resonance condition [see Eq. (1)] $\gamma_{R}(\hat{z})=\gamma_{R}(0) f_{R}(\hat{z})$ where $f_{R}(\hat{z})=\sqrt{\frac{1+K^{2}(\hat{z}) / 2}{1+K_{0}^{2} / 2}}$. Here $|\mathcal{E}|=|E| / \sqrt{4 \pi n_{0} \rho \gamma_{R}(0) m_{0} c^{2}}$ is the normalized amplitude of the electric field $E$.

We leave the $\hat{z}$-dependence in Eq. (2) while note that for untapered case, $f_{B}=f_{R}=1, \eta_{R}=0$ and $\mathcal{H}$ itself is a constant and independent of $\hat{z}$. From Hamilton's equations, we obtain the single-particle equations of motion for electron phase, energy deviation and the normalized electric field

$$
\begin{aligned}
& \frac{d \theta}{d \hat{z}}=\frac{\eta-\eta_{R}(\hat{z})}{f_{R}(\hat{z})} \\
& \frac{d \eta}{d \hat{z}}=-\frac{f_{B}(\hat{z})}{f_{R}(\hat{z})}\left(\mathcal{E} e^{i \theta}+\mathcal{E}^{*} e^{-i \theta}\right) \\
& \frac{\partial \mathcal{E}}{\partial \hat{z}}=\frac{f_{B}(\hat{z})}{f_{R}(\hat{z})}\left\langle e^{-i \theta}\right\rangle
\end{aligned}
$$

where the bracket $\langle\ldots\rangle$ denotes an ensemble average over the electron beam in the steady state (or a single bunch slice). The wave equation is obtained by taking the slowly varying envelope approximation. For simplicity we have neglected the index $j$. Note that there are a total of $\left(2 N_{e}+1\right)$ equations in the 1-D FEL system. The analysis below starts from the first saturation location. We remind that the theory does not distinguish the beginning of undulator tapering from the saturation location. Assuming $\Theta=\theta+\phi$, we have for the particle with the reference energy $\gamma_{R}(\hat{z})$,

$$
\frac{d \eta_{R}}{d \hat{z}}=-2 \frac{f_{B}(\hat{z})}{f_{R}(\hat{z})}|\mathcal{E}(\hat{z})| \cos \Theta_{R}
$$

where $\mathcal{E}=|\mathcal{E}| e^{i \phi}$ with $\phi$ the phase of the radiation field and $\Theta_{R}=\theta_{R}+\phi$. In order for the electron to have a decrease of energy at resonance, we require $\Theta_{R} \in[-\pi / 2, \pi / 2]$. For an untapered FEL, the reference phase is assumed to be fixed at $\Theta_{R}=-\pi / 2$. For particles near the resonant electron, i.e., $\theta=\theta_{R}+\delta \theta$, we can derive the small-amplitude synchrotron frequency as

$$
\Omega_{\mathrm{syn}}(\hat{z})=\left[-2 \frac{f_{B}(\hat{z})}{f_{R}^{2}(\hat{z})}|\mathcal{E}(\hat{z})| \sin \Theta_{R}\right]^{1 / 2} .
$$

Those near-resonant electrons will execute an oscillation or rotation in phase space with the synchrotron frequency $\Omega_{\text {syn }}$. It is straightforward to find the energy conservation from Eqs. (4) that

$$
|\mathcal{E}(\hat{z})|^{2}+\langle\eta\rangle=C_{1}
$$

In what follows we assume $C_{1}$ to be zero. This is validated for an initially monoenergetic unbunched electron beam and a low initial level of radiation [42]. The other constant of motion comes from adiabatic invariant of action variable. The action variable is defined as $I \equiv \oint d \eta d \theta$. If the change of fraction of undulator tapering is slow compared with the synchrotron oscillation period, the corresponding action variable, i.e., adiabatic invariant, can be still considered as a constant and is expressed as $\langle\mathcal{H}(\hat{z})\rangle / \Omega_{\text {syn }}(\hat{z})=C_{2}$. That is, we have

$\frac{\left\langle\eta-\eta_{R}\right\rangle^{2}}{2 \Omega_{\mathrm{syn}}(\hat{z}) f_{R}(\hat{z})}+\frac{2 f_{B}(\hat{z})}{\Omega_{\mathrm{syn}}(\hat{z}) f_{R}(\hat{z})}|\mathcal{E}(\hat{z})|\langle\sin (\theta+\phi)\rangle=C_{2}$.

Figure 1(a) shows the evolution of $C_{1}$ and $C_{2}$ for a typical tapered FEL process. In this example a constant resonant phase $\Theta_{R}=-80^{\circ}$ is assumed. The taper profile, shown in Fig. 1(b), follows that of Eq. (13) below. We see that the energy conservation [Eq. (7)] is well preserved and the single-particle Hamiltonian [Eq. (2)] is no longer a constant. However $C_{2}$ [Eq. (8)], under adiabatic approximation, serves as a passable invariant of motion with a negligible fractional deviation from the zero value. Note that evident synchrotron oscillations emerge after FEL saturation; therefore $C_{2}$ is only valid in the postsaturation regime.

Despite our primary focus of saturation dynamics, it deserves here to briefly summarize the universal
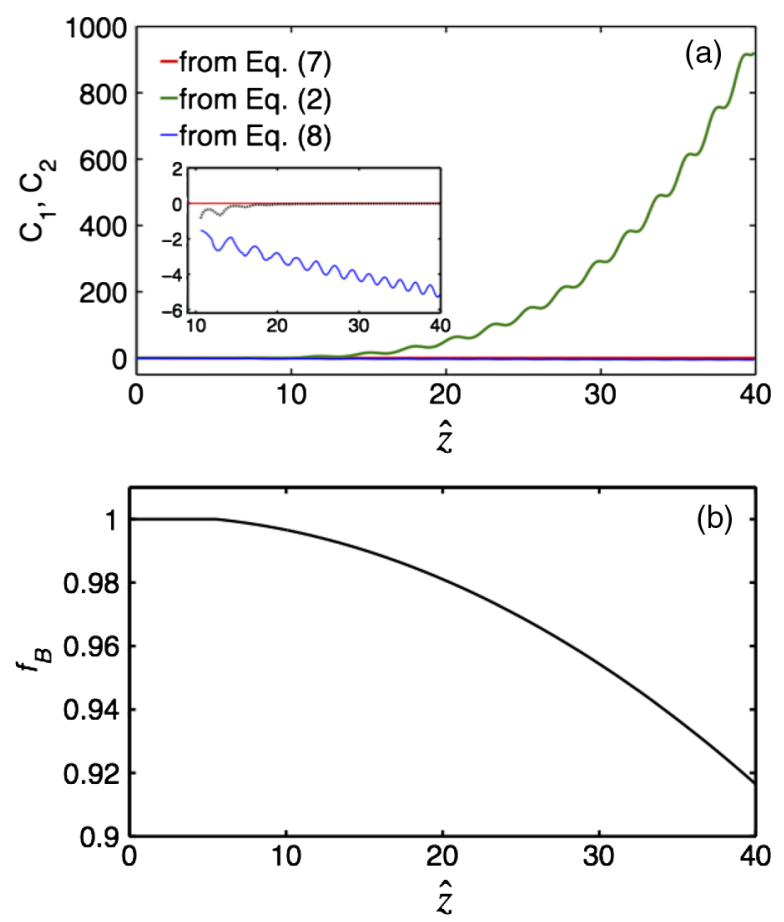

FIG. 1. Evolution of (a) the single-particle Hamiltonian $\langle\mathcal{H}(\hat{z})\rangle$ (green), $C_{1}$ (red) and $C_{2}$ (blue); (b) the taper profile $f_{B}(\hat{z})$. In the simulation the starting location of undulator tapering is set at $\hat{z}=5.5$ and the initial seed field $|\mathcal{E}(0)|=0.01$. The inset in (a) zooms in on $C_{1}$ and $C_{2}$ and the black dotted line indicates the ratio $C_{2} /\langle\mathcal{H}\rangle$. 
characteristics prior to the first saturation regime and use Eqs. (4) to obtain two important results. By defining the two collective variables, density bunching $b=\left\langle e^{-i \theta}\right\rangle$, energy bunching $p=\left\langle\eta e^{-i \theta}\right\rangle$, with the scaled main-signal field $\mathcal{E}(\hat{z})$, we can derive the third-order ordinary differential equation for $\mathcal{E}(\hat{z})$, which indicates the exponential growth in the collective FEL instability of the main signal [34]. Upon saturation, where $|\mathcal{E}(\hat{z})|^{2}=\mathcal{O}(1)$, we have, from Eq. (7) and the definition of $\eta,\langle\eta\rangle \leq-1$, which means that the mean electron energy begins to fall outside the $\rho$. Moreover, from the definition of the scaled field, the first-saturation power efficiency is estimated to be $P_{\text {sat }} / P_{\text {beam }}=|E|^{2} / 4 \pi n \gamma_{R 0} m c^{2} \approx \rho|\mathcal{E}|^{2} \sim \rho$. That is, in the untapered case the FEL efficiency is about $\rho$, usually smaller than $0.1 \%$ in $\mathrm{x}$-ray regime.

Aside from Eq. (7), the energy conservation can be expressed in another useful form. Let us decompose Eq. (4c) into the amplitude and phase components. We have

$$
\frac{d|\mathcal{E}|}{d \hat{z}}=\frac{f_{B}}{f_{R}}\langle\cos \Theta\rangle,
$$

and

$$
\frac{d \phi}{d \hat{z}}=-\frac{f_{B}}{|\mathcal{E}| f_{R}}\langle\sin \Theta\rangle .
$$

From Eqs. (5) and (9) and assuming $\langle\cos \Theta\rangle \approx \cos \Theta_{R}$, we have

$$
|\mathcal{E}(\hat{z})|^{2}=\left|\mathcal{E}\left(\hat{z}_{b}\right)\right|^{2}+\frac{1-f_{R}(\hat{z})}{\rho},
$$

for $\hat{z}>\hat{z}_{b}$, where the relation of $\eta_{R}=\left(f_{R}-1\right) / \rho$ has been used, $\hat{z}_{b}$ is the starting location of undulator tapering, and $\left|\mathcal{E}\left(\hat{z}_{b}\right)\right|$ is denoted as the normalized field amplitude at the starting location. The post-saturation tapered FEL efficiency can now be estimated to be $\rho|\mathcal{E}|^{2}=$ $\rho\left|\mathcal{E}\left(\hat{z}_{b}\right)\right|^{2}+1-f_{R}\left(\hat{z}_{f}\right)=\rho+\Delta \simeq \Delta$. Notice that the restrictive assumption of $\langle\cos \Theta\rangle \approx \cos \Theta_{R}$ indicates that the electrons are deeply trapped in the ponderomotive potential well. Under this situation through a proper undulator tapering the power efficiency can reach up to the level of the total taper ratio. In the following discussion we consider the constant resonant phase, i.e., $\Theta_{R}=$ constant. For the case with adiabatic undulator tapering, the increase of the main signal as a function of $\hat{z}$ can be obtained via Eq. (9)

$$
|\mathcal{E}(\hat{z})| \approx\left|\mathcal{E}\left(\hat{z}_{b}\right)\right|+\left(\hat{z}-\hat{z}_{b}\right) \cos \Theta_{R}
$$

assuming $f_{B} / f_{R}=\mathcal{O}(1)$.

Substituting into Eq. (5) with help of $\eta_{R}=\left(f_{R}-1\right) / \rho$, we have

$$
\begin{aligned}
f_{R}(\hat{z}) \approx & f_{B}(\hat{z}) \\
\approx & 1-2 \rho\left|\mathcal{E}\left(\hat{z}_{b}\right)\right| \cos \Theta_{R}\left(\hat{z}-\hat{z}_{b}\right) \\
& -\rho \cos ^{2} \Theta_{R}\left(\hat{z}-\hat{z}_{b}\right)^{2} .
\end{aligned}
$$

This is the derived taper profile based on the constant $\Theta_{R}$. Unlike the untapered case, where the radiation phase is linearly proportional to $\hat{z}$, the tapered radiation phase increases at a lower rate and scales logarithmically. This can be seen by integrating Eq. (10) over $\hat{z}$ assuming $f_{B}$ and $f_{R}$ vary slowly along $\hat{z}$ and $f_{B} / f_{R}=\mathcal{O}(1)$,

$\phi(\hat{z}) \approx \begin{cases}\phi\left(\hat{z}_{b}\right)-\frac{\sin \Theta_{R}}{\left|\mathcal{E}\left(\hat{z}_{b}\right)\right|}\left(\hat{z}-\hat{z}_{b}\right), & \Theta_{R}=-\frac{\pi}{2}, \\ \phi\left(\hat{z}_{b}\right)-\tan \Theta_{R} \ln \left[1+\frac{\cos \Theta_{R}}{\left|\mathcal{E}\left(\hat{z}_{b}\right)\right|}\left(\hat{z}-\hat{z}_{b}\right)\right], & -\frac{\pi}{2}<\Theta_{R} \leq 0\end{cases}$

Figure 2 demonstrates the evolution of the radiation amplitude and phase along $\hat{z}$. The logarithmic dependence can be Taylor expanded by employing $\ln (1+x) \approx$ $x-x^{2} / 2+-\cdots$. Figure 2(b) shows the linear and (up to) quadratic fittings to the untapered and tapered phase evolution, respectively. Through this numerical observation we will, for simplicity, assume the (up to) quadratic dependence of the radiation phase evolution along $\hat{z}$.
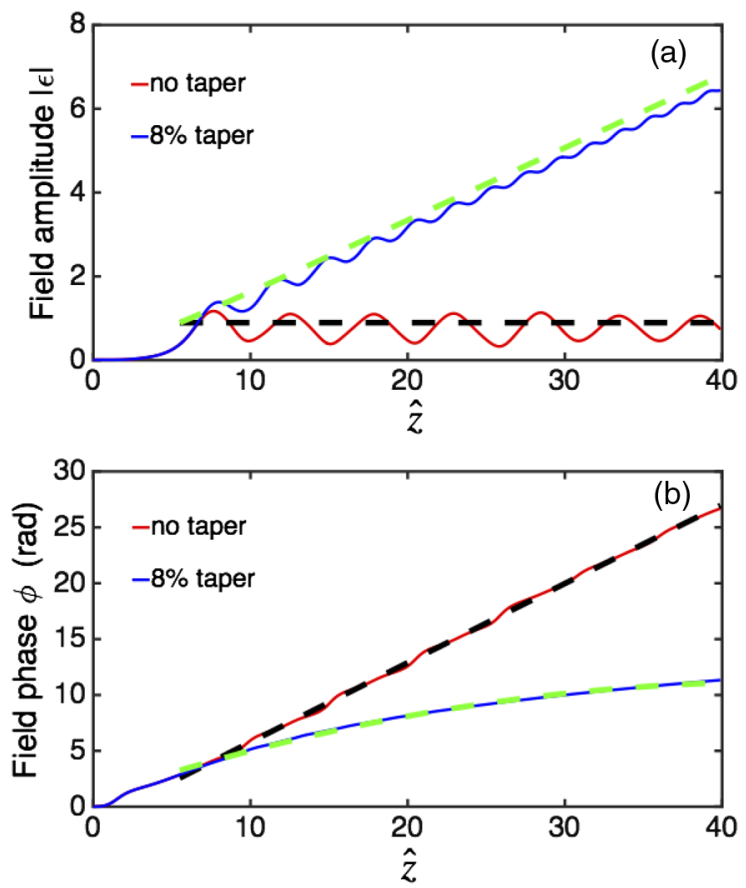

FIG. 2. Evolution of the radiation amplitude (a) and phase (b) along $\hat{z}$ for untapered and $8 \%$ tapered case. Dotted lines in (a) are obtained from Eq. (12) and those in (b) are fitted based on Taylor expansion of Eq. (14) to the first (linear, for no taper) and the second (quadratic, for $8 \%$ taper) orders. Relevant numerical parameters follow from Fig. 1. 
In this section we have already formulated the 1-D FEL equations, including the electron dynamics and the radiation field evolution. We will discuss in the next section the equilibrium state of the first saturation to derive several useful properties of electron beam for later use, and then in Sec. II C extend the 1-D field equation to axisymmetric 2-D case with inclusion of diffraction effect to study the effect on FEL saturation performance.

\section{B. Equilibrium solution and electron beam properties}

Below in this subsection we take advantage of Gluckstern et al. [42] to parametrize the radiation field solution based on the equilibrium solution. The equilibrium solution here is referred to as the solution evaluated at the starting location of the undulator tapering or the firstsaturation location. Again we remind that the theory does not distinguish the difference between the two locations. For the solution of the radiation field, we take the following form

$$
\mathcal{E}=(P+i Q) e^{i \Phi},
$$

where $\Phi \approx \kappa_{0}+\kappa_{1}\left(\hat{z}-\hat{z}_{0}\right)+\frac{\kappa_{2}}{2}\left(\hat{z}-\hat{z}_{0}\right)^{2}$. After the parametrization of $e^{i \Phi}, P$ and $Q$ are connected to the growth and the residual oscillation parts of the field amplitude. Here $\kappa_{1}$ and $\kappa_{2}$ further characterize the linear and quadratic dependence on $\hat{z}$ and $\kappa_{0}$ is a constant offset due to the fitting. We will ignore this offset in the following analysis. Through a proper choice of $\hat{z}_{0}$, which can be $\hat{z}_{b}$ but not necessary, $P$ can approach a linear dependence on $\hat{z}$ (or a constant for an untapered case) and $Q$ will oscillate about zero value, shown in Fig. 3. The coefficients $\kappa_{1}$ and $\kappa_{2}$ may vary for different choices of $\hat{z}_{0}$ to ensure the fitted $\Phi$ represents the field phase shown in Fig. 2. Here we note that a more rigorous treatment should impose an additional constraint on the amplitude and phase of the radiation field, i.e., the

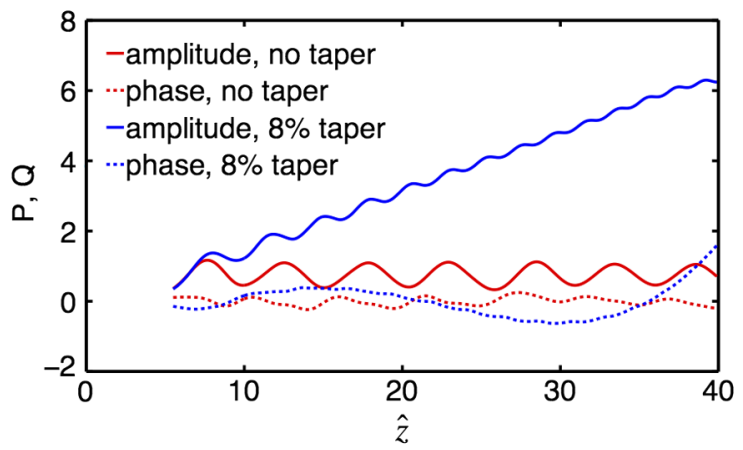

FIG. 3. Evolution of $P$ (solid lines) and $Q$ (dotted lines) for the untapered (red) and tapered (blue) cases. In obtaining $Q$ we choose $\hat{z}_{0}=\hat{z}_{b}=5.5$. The fitted coefficients are $\kappa_{1} \approx 0.714$, $\kappa_{2}=0$ for the untapered case, and $\kappa_{1} \approx 0.471, \kappa_{2} \approx-1.07 \times 10^{-2}$ for the $8 \%$ tapered case [see also Fig. 2(b)].
Kramers-Kronig relation. Here of our more interest is the evolution of field amplitude (or power) along $\hat{z}$; detailed account of phase contribution is overlooked in our analysis. In what follows, we adopt $P$, instead of $|\mathcal{E}|$, as the field amplitude (not to be confused with the radiation power $P_{\text {rad }}$ or electron beam power $P_{\text {beam }}$ ).

Let us introduce the variable $\beta$ for the subsequent analysis, $\beta=\theta-\theta_{R}-\Phi$, which represents the displaced electron phase and $\left\langle\beta^{\prime}\right\rangle \approx 0$ in the equilibrium [42]. Then Eqs. (4) can be rewritten as

$\frac{d \beta}{d \hat{z}}=\frac{d\left(\theta-\theta_{R}\right)}{d \hat{z}}-\kappa_{1}$

$\frac{d \beta^{\prime}}{d \hat{z}}=-2 \frac{f_{B}(\hat{z})}{f_{R}^{2}(\hat{z})}\left(P \cos \beta-Q \sin \beta-P \cos \Theta_{R}\right)+\kappa_{2}$

$\frac{\partial P}{\partial \hat{z}}=\kappa_{1} Q+\frac{f_{B}(\hat{z})}{f_{R}(\hat{z})}\langle\cos \beta\rangle$

$\frac{\partial Q}{\partial \hat{z}}=-\kappa_{1} P-\frac{f_{B}(\hat{z})}{f_{R}(\hat{z})}\langle\sin \beta\rangle$

where the prime here and in what follows denotes the derivative with respect to $\hat{z}$.

The two constants of motion, Eqs. (7) and (8), can also be expressed in the following forms,

$$
P^{2}+Q^{2}+f_{R}\left[\left\langle\beta^{\prime}\right\rangle-\kappa_{1}\right]+\eta_{R}=0,
$$

and

$\frac{f_{R}}{\Omega_{\text {syn }}}\left\{\frac{\left\langle\beta^{\prime 2}\right\rangle}{2}-\kappa_{1}\left\langle\beta^{\prime}\right\rangle+\frac{\kappa_{1}^{2}}{2}+2 \frac{f_{B}}{f_{R}^{2}}(P\langle\sin \beta\rangle+Q\langle\cos \beta\rangle)\right\} \approx 0$.

In the presence of undulator tapering, we have known the functions $P$ and $Q$ in Eq. (15) can be approximately expressed as

$$
\begin{aligned}
P(\hat{z}) & \approx\left|\mathcal{E}\left(\hat{z}_{b}\right)\right|+\left(\hat{z}-\hat{z}_{b}\right) \cos \Theta_{R}, \\
Q & \approx 0, \quad Q^{\prime} \approx 0 .
\end{aligned}
$$

The two constants of motion can be further simplified to be

$$
\kappa_{1}=\frac{P^{2}+\eta_{R}}{f_{R}},
$$

and

$$
\left\langle\beta^{2}\right\rangle=4 \kappa_{1} P^{2}-\kappa_{1}^{2} .
$$

It has been known that the equilibrium (or unperturbed) electron beam phase space distribution satisfies the Vlasov 
equation. The unperturbed solution can always be expressed in an arbitrary function of the constant(s) of motion [49]. From the above analysis we have made the choice of using the action variable as an invariant. In what follows, by taking advantage of the conclusion by Gluckstern et al. [42], we adopt the Boltzmann-type distribution as the unperturbed solution. Therefore it can be written in the form

$$
f_{\mathrm{BZM}}=\mathcal{N} e^{-\alpha \tilde{I}\left(\beta, \beta^{\prime} ; \hat{z}\right)},
$$

where $\mathcal{N}$ is the normalization coefficient such that $\left\langle f_{\mathrm{BZM}}\right\rangle \equiv \int_{0}^{2 \pi} \int_{-\infty}^{\infty} d \beta d \beta^{\prime} f_{\mathrm{BZM}}\left(\beta, \beta^{\prime}\right)=1$, and $\alpha$ is a measure of the particle spread in the ponderomotive potential well [43]. From Eq. (18) we take $\tilde{I}\left(\beta, \beta^{\prime} ; \hat{z}\right)=$ $\frac{f_{R}}{2 \Omega_{\mathrm{syn}}}\left(\beta^{\prime}-\kappa_{1}\right)^{2}+\frac{2 f_{B}}{\Omega_{\mathrm{syn}} f_{R}} P \sin \beta$. For convenience we also define $\chi=f_{B} / \Omega_{\text {syn }}$, and $\mathcal{K}=\alpha f_{R} / \Omega_{\text {syn }}$. Several relevant quantities can be evaluated, including the normalization coefficient

$$
\mathcal{N}=\frac{\sqrt{\mathcal{K}}}{(2 \pi)^{3 / 2} I_{0}(2 \alpha \chi P)} .
$$

On the right-hand side of Eqs. (16c) and (16d), the bunching parameters can also be evaluated

$$
\langle\sin \beta\rangle=-\frac{I_{1}(2 \alpha \chi P)}{I_{0}(2 \alpha \chi P)} \approx-\alpha \chi P \equiv-\bar{s},
$$

where $I_{0}$ and $I_{1}$ are respectively the zeroth- and first-order modified Bessel function of the first kind, and

$$
\langle\cos \beta\rangle=\cos \Theta_{R}, \quad\left\langle\frac{f_{B}}{f_{R}} \cos \beta-\cos \Theta_{R}\right\rangle \approx 0,
$$

and

$$
\left\langle\left(\beta^{\prime}-\kappa_{1}\right)^{2}\right\rangle=\mathcal{K}^{-1}, \quad\left\langle\beta^{2}\right\rangle=\mathcal{K}^{-1}+\kappa_{1}^{2} .
$$

Up to now we have obtained several relevant parameters for the electron beam expressed in terms of $P$ and $Q$, based on the arguments in Eqs. (15) and (22). We will apply these results for our subsequent analysis.

\section{Extension to transverse finite-size beams}

In this subsection we will extend the 1-D wave equation formulated in Sec. II A to axisymmetric 2-D equation including the radial dependence of the radiation field. The analysis will include the diffraction effect and the guiding properties formed by the bunched electron beam through the FEL process. The electron beam now features a finite transverse size but the betatron oscillation and the angular divergence (or, transverse emittance) are neglected for simplicity. In reality due to the external strong focusing imposed on the electron beam, the betatron motion and the nonzero angular spread (or, finite emittance) should be taken into account. The radiation diffraction effect is included in the transverse Laplacian operator and the wave equation can be written as

$$
\frac{\partial \mathcal{E}}{\partial \hat{z}}-i \nabla_{\perp}^{2} \mathcal{E}=\frac{f_{B}(\hat{z})}{f_{R}(\hat{z})} U(\hat{r})\left\langle e^{-i \theta}\right\rangle,
$$

where $U(\hat{r})$ is the transverse electron beam profile and the scaled radius $\hat{r}=\sqrt{4 \rho k_{u} k_{R}} r . \nabla_{\perp}^{2}=\hat{r}^{-1} \partial / \partial \hat{r}(\hat{r} \partial / \partial \hat{r})$. In the absence of the right hand side term, Eq. (27) becomes the paraxial Helmholtz equation and the solution can be typically parametrized by the Gaussian beam [50]. The presence of nonzero term on the right-hand side of Eq. (27) acts as an external medium. This medium, due to electron beam microbunching, leads to an effective index of refraction greater than unity [27]. Now, let us write Eq. (27) in terms of the aforementioned $P$ and $Q$,

$$
\begin{aligned}
& \frac{\partial P}{\partial \hat{z}}+\nabla_{\perp}^{2} Q=\kappa_{1} Q+\frac{f_{B}(\hat{z})}{f_{R}(\hat{z})} U(\hat{r})\langle\cos \beta\rangle \\
& \frac{\partial Q}{\partial \hat{z}}-\nabla_{\perp}^{2} P=-\kappa_{1} P-\frac{f_{B}(\hat{z})}{f_{R}(\hat{z})} U(\hat{r})\langle\sin \beta\rangle .
\end{aligned}
$$

From Eqs. (28b) and (19) we have

$$
\kappa_{1} P-\nabla_{\perp}^{2} P=-\frac{f_{B}(\hat{z})}{f_{R}(\hat{z})} U(\hat{r})\langle\sin \beta\rangle .
$$

The two constants of motion, Eqs. (17) and (18), can now be integrated over $\hat{r}$ and become

$$
\int_{0}^{\infty} \hat{r} d \hat{r}\left\{P^{2}+Q^{2}+U(\hat{r})\left[f_{R}\left(\left\langle\beta^{\prime}\right\rangle-\kappa_{1}\right)+\eta_{R}\right]\right\}=0,
$$

and

$$
\begin{gathered}
\int_{0}^{\infty} \hat{r} d \hat{r}\left\{\frac{f_{R} U(\hat{r})}{\Omega_{\mathrm{syn}}}\left\langle\left(\beta^{\prime}-\kappa_{1}\right)^{2}\right\rangle-\frac{2}{\Omega_{\mathrm{syn}}}\left[P Q^{\prime}-P^{\prime} Q\right.\right. \\
\left.\left.+\kappa_{1}\left(P^{2}+Q^{2}\right)+\left(\nabla_{\perp} P\right)^{2}+\left(\nabla_{\perp} Q\right)^{2}\right]\right\}=0 .
\end{gathered}
$$

Using Eq. (19), we can simplify the above two equations

$$
\int \hat{r} d \hat{r} P^{2}(\hat{r} ; \hat{z})=\left(f_{R} \kappa_{1}-\eta_{R}\right) \int \hat{r} d \hat{r} U(\hat{r}),
$$

and

$$
\int \hat{r} d \hat{r} U(\hat{r})\left\langle\left(\beta^{\prime}-\kappa_{1}\right)^{2}\right\rangle=\frac{2}{f_{R}} \int \hat{r} d \hat{r}\left[\kappa_{1} P^{2}+\left(\nabla_{\perp} P\right)^{2}\right] .
$$


Now we can solve the field equation, together with the constants of motion in a given transverse electron beam profile. Let us consider the simplest case, i.e., the uniform stepped profile with the form

$$
U(\hat{r})= \begin{cases}1, & \hat{r} \leq \hat{R} \\ 0, & \hat{r}>\hat{R}\end{cases}
$$

where $\hat{R}=\sqrt{4 \rho k_{u} k_{R}} R$ is the boundary of the transverse electron beam density profile. Inserting into Eq. (28b) gives

$$
\begin{aligned}
{\left[\nabla_{\perp}^{2}+\alpha \chi \frac{f_{B}(\hat{z})}{f_{R}(\hat{z})}-\kappa_{1}\right] P } & =0, \text { inside the beam } \\
\left(\nabla_{\perp}^{2}-\kappa_{1}\right) P & =0, \text { outside the beam. }
\end{aligned}
$$

The continuity conditions of $P$ and $d P / d \hat{r}$ at the beam boundary leads to the following constraint,

$$
y J_{1}(y) K_{0}(x)=x J_{0}(y) K_{1}(x),
$$

where $x=\sqrt{\kappa_{1}} \hat{R}, \quad y=\mu \hat{R}$, and $\mu=\sqrt{\alpha \chi f_{B} / f_{R}-\kappa_{1}}$. Equation (36) sets up a constraint on $x$ and $y$, i.e., $\alpha$ and $\kappa_{1}$. The explicit expression of the radiation field solution can now be written as

$$
P(\hat{r} ; \hat{z})= \begin{cases}\mathcal{A} J_{0}(\mu \hat{r}), & \hat{r} \leq \hat{R} \\ \mathcal{A} \frac{J_{0}(y)}{K_{0}(x)} K_{0}\left(\sqrt{\kappa_{1}} \hat{r}\right), & \hat{r}>\hat{R}\end{cases}
$$

where $\mathcal{A}$ is the on-axis field amplitude, $J_{0}$ and $K_{0}$ are the zeroth order ordinary Bessel function and the modified Bessel function of the second kind, respectively. Throughout the analysis we consider the single-mode configuration, i.e., the $\mathrm{TEM}_{00}$ mode. This is justified that only such a mode can exist after the exponential growth and proceed in the postsaturation regime. The resultant field solution needs to satisfy the aforementioned two constants of motion. By substituting Eq. (37) into Eqs. (32) and (33) we obtain

$f_{R} \kappa_{1}-\eta_{R}=\mathcal{A}^{2}\left\{J_{0}^{2}(y)+J_{1}^{2}(y)+\frac{J_{0}^{2}(y)}{K_{0}^{2}(x)}\left[K_{1}^{2}(x)-K_{0}^{2}(x)\right]\right\}$,

and

$$
\kappa_{1}^{2}=2 \alpha \chi \frac{f_{B}}{f_{R}} \mathcal{A}^{2}\left[J_{0}^{2}(y)+J_{1}^{2}(y)\right]-\frac{1}{2 \mathcal{K}},
$$

where $\chi=f_{B} / \Omega_{\mathrm{syn}}$, and $\mathcal{K}=\alpha f_{R} / \Omega_{\mathrm{syn}}$.

In the case of the uniform electron beam profile, Eqs. (36), (38), and (39) will be numerically solved for $\kappa_{1}, \alpha$, and $\mathcal{A}$ [i.e., $P(\hat{r}=0)$ ]. Of our particular interest the combined term $\left(f_{R} \kappa_{1}-\eta_{R}\right)$ represents the FEL power efficiency,

$$
f_{R} \kappa_{1}-\eta_{R}=\frac{\int \hat{r} d \hat{r} P^{2}(\hat{r} ; \hat{z})}{\int \hat{r} d \hat{r} U(\hat{r})}
$$

The electron energy spread will increase during the saturated FEL process and can be characterized by the scaled energy spread

$$
\begin{aligned}
\left\langle\left(\eta-\eta_{R}\right)^{2}\right\rangle & =\int_{0}^{2 \pi} d \theta \int_{-\infty}^{\infty} d \eta\left(\eta-\eta_{R}\right)^{2} f_{\mathrm{BZM}} \\
& =\int_{0}^{2 \pi} d \beta \int_{-\infty}^{\infty} d \beta^{\prime}\left|J\left(\frac{\partial(\theta, \eta)}{\partial\left(\beta, \beta^{\prime}\right)}\right)\right|\left(\beta^{\prime}-\kappa_{1}\right)^{2} f_{\mathrm{BZM}} \\
& =\frac{\Omega_{\mathrm{syn}}}{\alpha}
\end{aligned}
$$

where the Jacobian is due to the coordinate transformation, $\left|J\left(\frac{\partial(\theta, \eta)}{\partial\left(\beta, \beta^{\prime}\right)}\right)\right|=f_{R}$ [see also Eq. (16)].

The second example allowing analytical solutions refers to the bounded parabolic profile,

$$
U(\hat{r})= \begin{cases}1-\Upsilon^{2} \hat{r}^{2}, & \hat{r} \leq \hat{R} \\ 0, & \hat{r}>\hat{R}\end{cases}
$$

where $\Upsilon \equiv k_{1} / \hat{R}$. Here $k_{1}$ is a free parameter to characterize the cutoff of the profile distribution.

Similarly, inserting Eq. (42) into Eq. (28b) gives us the governing equation for the radiation field inside and outside the electron beam

$$
\left[\nabla_{\perp}^{2}+\frac{\alpha \chi f_{B}}{f_{R}}\left(1-\Upsilon^{2} \hat{r}^{2}\right)-\kappa_{1}\right] P(\hat{r})=0, \text { inside the beam }
$$

$$
\left(\nabla_{\perp}^{2}-\kappa_{1}\right) P(\hat{r})=0 \text {, outside the beam. }
$$

Notice that Eq. (43b) is identical to Eq. (35b). For convenience of solving Eq. (43a), we define the shorthand notation for the quantity $\delta^{2}=\frac{\alpha \chi f_{B}}{f_{R}} \Upsilon^{2}$. Then Eq. (43a) becomes

$$
\left(\frac{d^{2}}{d \hat{r}^{2}}+\frac{1}{\hat{r}} \frac{d}{d \hat{r}}+\mu^{2}-\delta^{2} \hat{r}^{2}\right) P(\hat{r})=0 .
$$

The solution to the above equation takes the form of the confluent hypergeometric function [9,51]. Matching the radiation field and its derivative at the beam boundary leads to a similar matching equation to Eq. (36),

$$
\begin{aligned}
& \delta \hat{R}^{2} K_{0}(x)\left[2 \epsilon_{1} F_{1}\left(\epsilon+1,2 ; \delta \hat{R}^{2}\right)-{ }_{1} F_{1}\left(\epsilon, 1 ; \delta \hat{R}^{2}\right)\right] \\
& \quad+x K_{1}(x){ }_{1} F_{1}\left(\epsilon, 1 ; \delta \hat{R}^{2}\right)=0
\end{aligned}
$$

where $\epsilon=1 / 2-\mu^{2} / 4 \delta$.

Note that we have used the relation $d_{1} F_{1}\left(\epsilon, 1 ; \delta \hat{r}^{2}\right) / d \hat{r}=$ $2 \epsilon \delta \hat{r}_{1} F_{1}\left(\epsilon+1,2 ; \delta \hat{r}^{2}\right)$. The general expression of the field solution can be expressed as 
$P(\hat{r} ; \hat{z})= \begin{cases}\mathcal{A} e^{-\delta \hat{r}^{2} / 2}{ }_{1} F_{1}\left(\epsilon, 1 ; \delta \hat{r}^{2}\right), & \hat{r} \leq \hat{R} \\ \mathcal{A} e^{-\delta \hat{R}^{2} / 2}{ }_{1} F_{1}\left(\epsilon, 1 ; \delta \hat{R}^{2}\right) \frac{K_{0}\left(\sqrt{\kappa_{1}} \hat{r}\right)}{K_{0}(x)}, & \hat{r}>\hat{R} .\end{cases}$

A special case of the bounded parabolic beam being reduced to be the uniform one, where $\delta \rightarrow 0$ (or $\Upsilon \rightarrow 0$ ), can be retrieved by taking advantage of the relation $\lim _{\delta \rightarrow 0}\left[{ }_{1} F_{1}\left(\epsilon, 1 ; \delta \hat{R}^{2}\right)\right]=J_{0}(\mu \hat{R})$. It is clear that Eq. (45) can be reduced to Eq. (36). Consistency requires that the two constants of motion must be satisfied by the radiation field solution. Before proceeding, let us define the following two functions

$$
\mathcal{F}_{1}(\epsilon)=\int_{0}^{1} e^{-t}\left[{ }_{1} F_{1}(\epsilon, 1 ; t)\right]^{2} d t,
$$

and

$$
\mathcal{F}_{2}(\epsilon)=\int_{0}^{1} t e^{-t}\left[{ }_{1} F_{1}(\epsilon, 1 ; t)\right]^{2} d t
$$

Note that the two functions depend only on $\epsilon$. For reference their functional behaviors are shown in Fig. 4.

The two constraints on the field solution can now be expressed in terms of $\mathcal{F}_{1}$ and $\mathcal{F}_{2}$ as

$$
\begin{aligned}
f_{R} \kappa_{1}-\eta_{R}= & \frac{\mathcal{A}^{2}}{\left(1-\frac{1}{2} \Upsilon^{2} \hat{R}^{2}\right)}\left\{\frac{\mathcal{F}_{1}(\epsilon)}{\delta \hat{R}^{2}}\right. \\
& \left.+e^{-\delta \hat{R}^{2}}\left(\frac{K_{1}^{2}(x)}{K_{0}^{2}(x)}-1\right)\left[{ }_{1} F_{1}\left(\epsilon, 1 ; \delta \hat{R}^{2}\right)\right]^{2}\right\},
\end{aligned}
$$

and

$$
\kappa_{1}^{2}=\frac{f_{B}}{f_{R}^{2}} \frac{2 \alpha \chi \mathcal{A}^{2}}{\delta \hat{R}^{2}\left(1-\frac{1}{2} \Upsilon^{2} \hat{R}^{2}\right)}\left[\mathcal{F}_{1}(\epsilon)-\frac{\Upsilon^{2}}{\delta} \mathcal{F}_{2}(\epsilon)\right]-\frac{1}{2 \mathcal{K}} .
$$

Similarly, Eq. (45), together with Eqs. (49) and (50), should be solved for $\kappa_{1}, \alpha, \mathcal{A}$, provided the remaining parameters are given.

In this subsection we have analytically obtained the radiation field profile in the presence of electron beam for

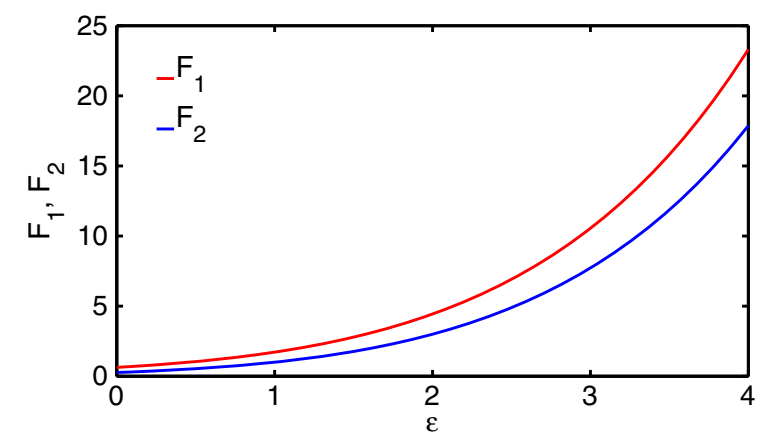

FIG. 4. Behavior of $\mathcal{F}_{1}$ and $\mathcal{F}_{2}$ defined in Eqs. (47) and (48). transverse uniform stepped and bounded parabolic profiles. Three parameters, governed by the FEL process, are subject to the functional form of the field distribution and two constants of motion. They include the measure of electron trapping in the ponderomotive potential well, the power enhancement in the postsaturation regime, and the on-axis radiation field amplitude. Before ending this section, we comment that the validity of the above analysis is based on the assumption that the bunched electrons are not significantly lost in the ponderomotive potential well (or the phase space bucket). In Sec. III we will numerically solve the three parameters to obtain the self-consistent results and discuss their scaling properties on the taper ratios and the transverse electron beam sizes.

\section{NUMERICAL RESULTS}

In this section we will first illustrate the radiation field solution derived from Sec. II by numerically solving the matching equations with constants of motion as constraints. Of our particular interest are dependencies of the resultant radiation field solution on the transverse beam size and on the taper ratio, i.e., the scaling properties. Then we qualitatively compare our findings using a full 3-D numerical simulation GENESIS [52].

\section{A. Scaling properties}

Let us begin with the untapered case. Figure 5 shows that power efficiency, increase of the scaled energy spread, and the half width at half maximum (HWHM) of the radiation field profile for an untapered case. In Fig. 5(a), we can see that the output efficiency reaches a saturation value $\sim 0.1 \%$ when the scaled transverse electron beam size is larger than 4. Note that such a value is close to the Pierce parameter of the short-wavelength high-gain FEL. Let us take the Linac Coherent Light Source (LCLS) like parameters as a practical example, $K_{0}=3.5, \lambda_{u}=3 \mathrm{~cm}$, and $\lambda_{R}=$ $2.755 \AA$ (or $4.5 \mathrm{keV}$ photon energy), which correspond to the reference electron energy $10 \mathrm{GeV}$ with a typical bunch current level $3 \mathrm{kA}$. A typical transverse electron beam size is about $20 \mu \mathrm{m}$. The Pierce parameter $\rho$ is about $1.5 \times 10^{-3}$. This corresponds to the scaled electron beam radius $\hat{R}=\sqrt{4 \rho k_{u} k_{R}} R \approx 3.4$, and the first saturation power is at the level of $10 \mathrm{GW}$. When the transverse beam size is increasing, the result will approach the 1-D limit. In addition to the power efficiency, the induced energy spread of the electron beam, shown in Fig. 5(b), will increase as well when the beam-wave interaction is improved. Inserting the numerically obtained $\kappa_{1}, \alpha$ and $\mathcal{A}$ into Eqs. (37) and (47) gives the resultant radiation field profile. The effective width can be characterized as the half width at half maximum (HWHM), indicating the effectiveness of the guided transverse mode.

Having illustrated the untapered case, let us study how the saturated power efficiency, the induced energy spread, 

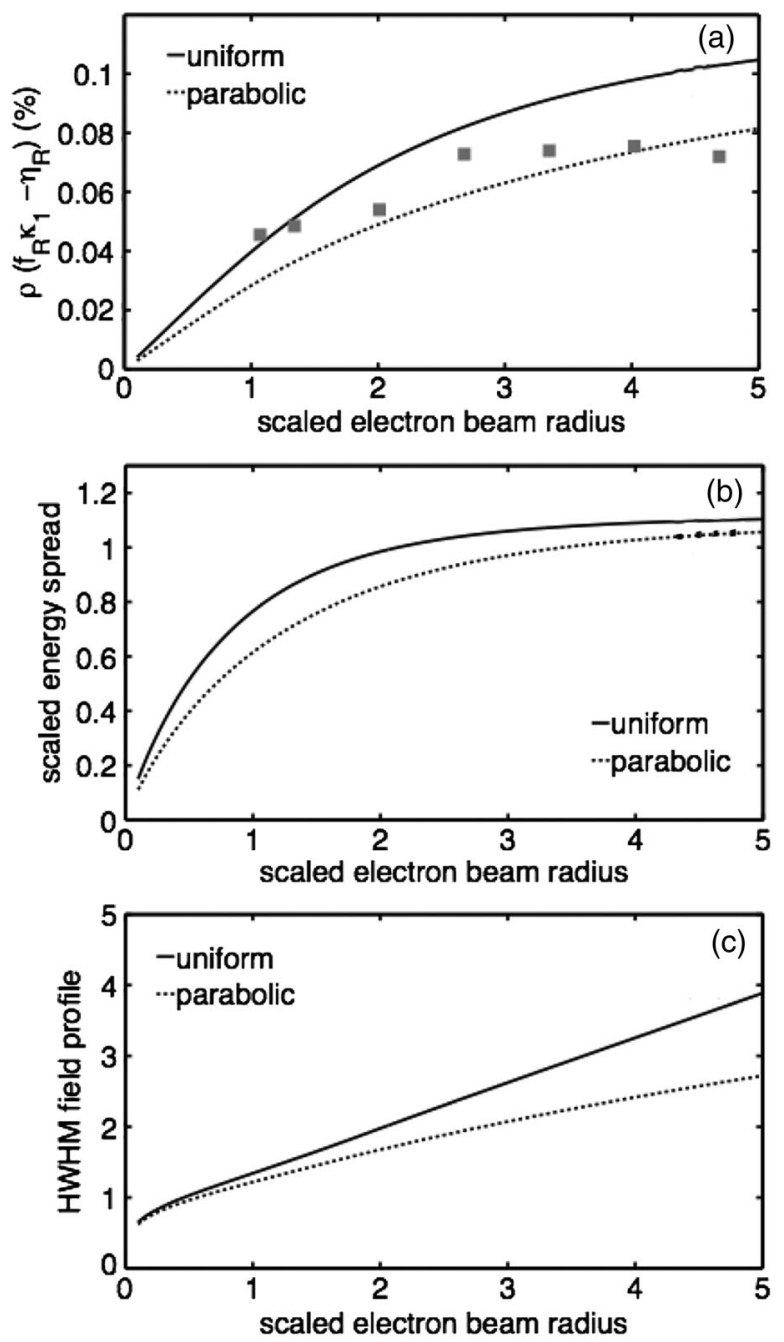

FIG. 5. Dependence of the power efficiency (a), scaled energy spread (b), and the half width of the radiation field profile (c) on the scaled transverse electron beam radius for an untapered case $\left(\Theta_{R}=-\pi / 2\right)$. The dots in (a) are obtained from 3-D numerical simulation results GENESIS.

and the effective width of the radiation field profile are affected by the undulator tapering. Figures 6(a) and 6(b) shows the dependence of the power efficiency on the scaled transverse electron beam size and the taper ratio, respectively. Unlike Fig. 5(a), the tapered power efficiency has very weak dependence on the transverse electron beam size. Going from $\hat{R}=0.1$ to $\hat{R}=5$ the theoretical predictions [Eqs. (38) and (49)] give only $0.02 \%$ and $0.01 \%$ difference for transverse uniform and parabolic distribution, respectively, for the case of $1 \%$ taper ratio. The difference becomes even smaller for $8 \%$ taper ratio. The dots in Fig. 6(a) are obtained from the full 3-D numerical simulation GENESIS, where we can see both the theoretical predictions and the numerical results match well for the untapered and 1\% taper cases. For $8 \%$ taper case, there appears a systematic deviation between the theoretical and simulation results. However the independence of the
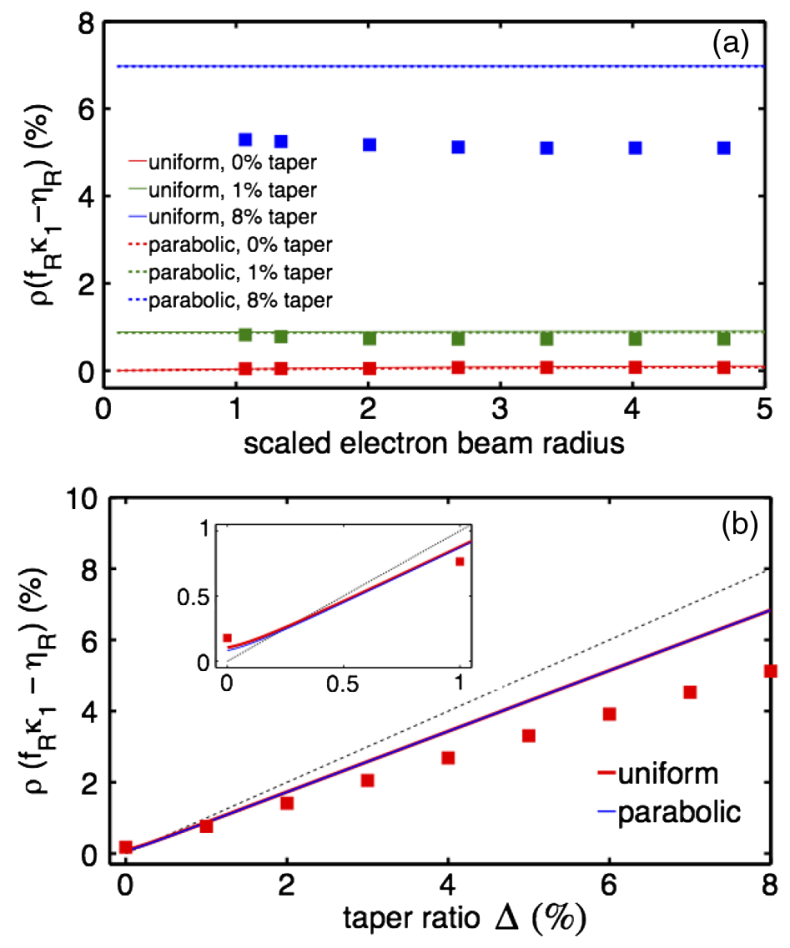

FIG. 6. The dependence of the power efficiency on the scaled transverse electron beam radius (a) for three specific taper ratios (untapered, $1 \%$ and $8 \%$ taper ratios), and the dependence on the taper ratio (b) for the uniform stepped and the bounded parabolic $\left(k_{1}=1\right)$ electron transverse beam profile. It can be seen that the power efficiency is almost independent of $\hat{R}$, although (b) is plotted at $\hat{R}=3$. The dots in (a,b) indicate the full 3-D numerical simulation results from GENESIS for the electron transverse uniform distribution. The inset in (b) shows the crossing of the calculated power efficiency to the $y=x$ dotted line.

transverse electron beam size is shown. The deviation can be attributed to the idealization we imposed for the electron beam transverse motion. Note that in the theoretical formulation we have ignored the betatron oscillation and the finite angular divergence of the electron beam, i.e., the finite emittance effect. By looking at another dimension of the power efficiency dependence, the power extraction efficiency is now almost linearly dependent on the taper ratio, as shown in Fig. 6(b). It is interesting to note that when the taper ratio approaches to zero, i.e., the untapered case, the power efficiency reaches a constant close to the FEL or Pierce parameter $\rho$. In Fig. 6(b) the dotted line denotes the case where we have discussed in Sec. II A [the text below Eq. (11)]. It is further confirmed here that in the beam-wave matched case the resultant power efficiency can be only slightly smaller but close to the total taper ratio. In view of Fig. 6, we remind that the above argument of power efficiency scaling does not apply to any arbitrarily large transverse beam size and to any level of taper ratio. The aforementioned discussion assumes negligible electron detrapping. The longitudinal phase space bucket (or the ponderomotive potential well) corresponding to the outer 
transverse portion of an electron beam can become smaller than that to the on-axis of the beam, due to the weaker and weaker radiation field amplitude, and this may give rise to electron detrapping. In that situation one can expect that in Fig. 6(b) the calculated power efficiency will saturate and be bent downward at an even larger taper ratio. Since the above analysis places emphasis on the core interaction between the electron beam and the radiation field, the detrapping is beyond the scope of our discussion.

Figures 7 and 8 depict the induced energy spread and the effective width of the radiation field during the FEL saturation process in terms of the transverse electron beam size and the level of undulator tapering. Above a certain transverse beam size the beam-wave interaction will gradually reach an equilibrium. In Fig. 7(b) as the taper ratio increases up to a level, at which it can effectively capture most of the electron energy loss, the electrons in the phase space (or in the ponderomotive bucket) will be further trapped, i.e., corresponding to smaller $\alpha$ [see Eqs. (22) and (41)]. The more effective beam-wave interaction then leads to the increase of the induced energy spread.

In addition to the above scaling properties, we find that in the presence of undulator tapering more of the enhanced radiation field intensity is contributed from outside of the transverse electron beam than from inside of it. Inserting the numerically obtained solutions for $\kappa_{1}, \alpha$, and $\mathcal{A}$ into the field expressions, Eqs. (37) and (47) for uniform and
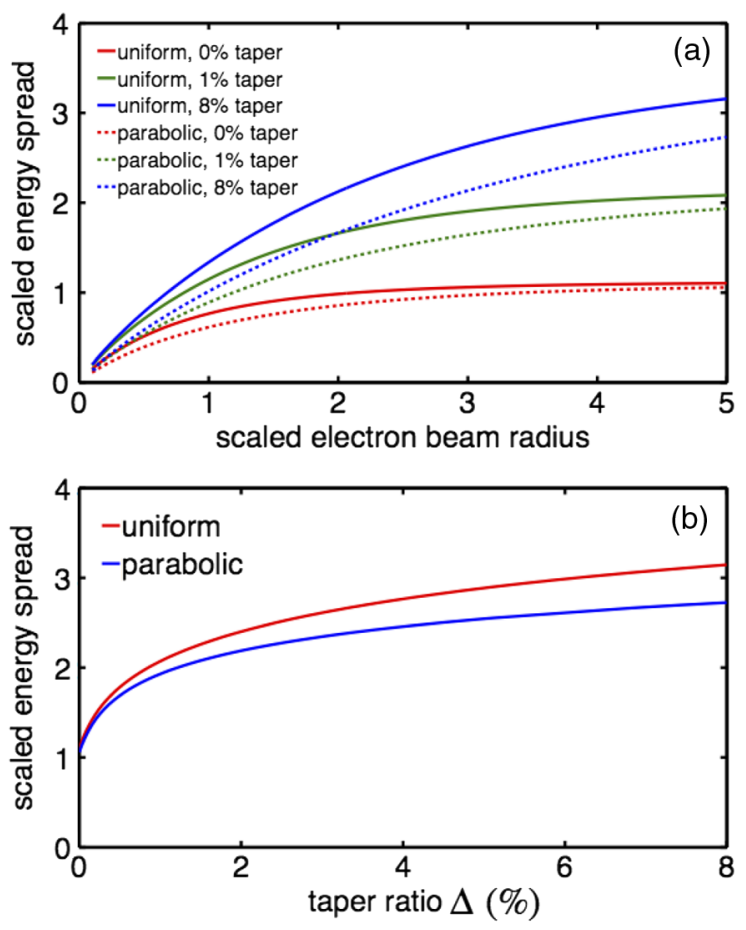

FIG. 7. The dependence of the scaled energy spread on the transverse electron beam radius (a) for three specific taper ratios (untapered, $1 \%$ and $8 \%$ taper ratios), and the dependence on the taper ratio (b) for the uniform stepped and the bounded parabolic $\left(k_{1}=1\right)$ electron transverse beam profile.
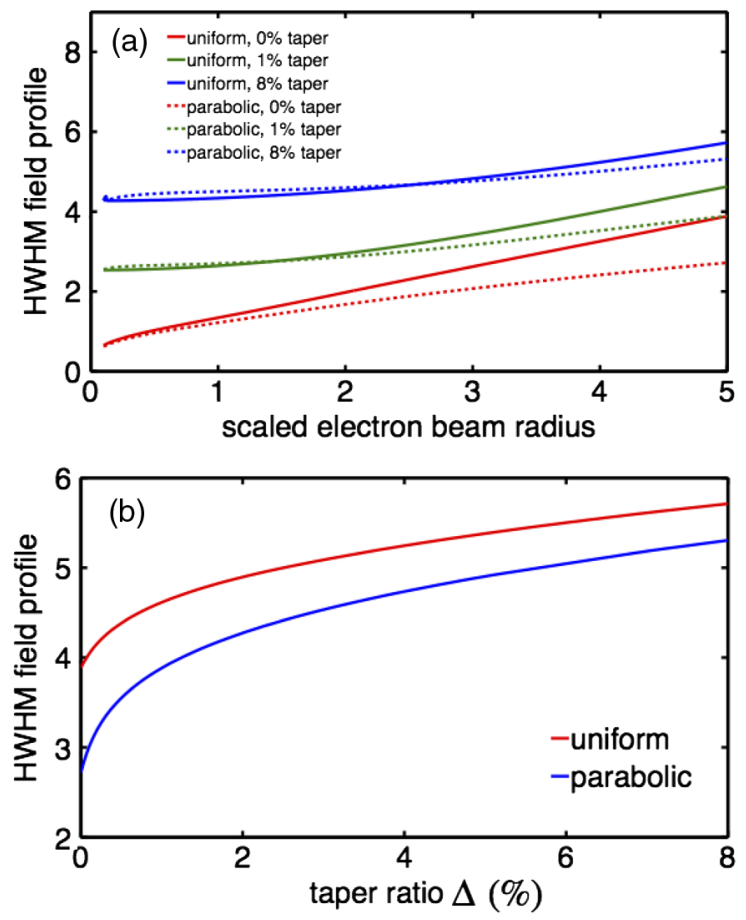

FIG. 8. The dependence of the size (half width at half maximum) of radiation field profile on the transverse electron beam radius (a) for three specific taper ratios (untapered, $1 \%$ and $8 \%$ taper ratios), and the dependence on the taper ratio (b) for the uniform stepped and the bounded parabolic $\left(k_{1}=1\right)$ electron transverse beam profile.

parabolic beam profiles, we can obviously see the distinction of the resultant radiation profiles between the untapered and tapered cases. Figure 9 illustrates the normalized field profiles for two different transverse beam sizes $(\hat{R}=2$ and 4$)$ of the uniform and parabolic profiles in the untapered and $8 \%$ tapered cases. This observation is also reflected in Fig. 8(b).

\section{B. Three-dimensional numerical simulation}

The above analysis is based on extension of 1-D to 2-D axisymmetric FEL formalism. In this subsection we also illustrate the dynamics of the radiation field profile by using the full 3-D time-independent simulation code GENESIS [52]. This numerical illustration enables us to see a clear picture how the spatial evolution of the radiation field intensity in both transverse and longitudinal dimensions, and the impact of undulator tapering. In the numerical simulation the electron beam transverse profile is set uniform round with the half width $\approx 28 \mu \mathrm{m}$ in $x$ and $y$ (or $\approx 40 \mu \mathrm{m}$ in $r$ ) and the total length of the undulator is assumed $70 \mathrm{~m}$ with $\lambda_{u}=2.6 \mathrm{~cm}$. The corresponding radiation wavelength is $3.1 \AA$. The first saturation power $\approx 22 \mathrm{GW}$ is matched at the beam waist with the input electron beam. The transverse domain in the numerical setup extends from $-0.8 \mathrm{~mm}$ to $+0.8 \mathrm{~mm}$ with a total number of $451 \times 451$ grid points to avoid possible 

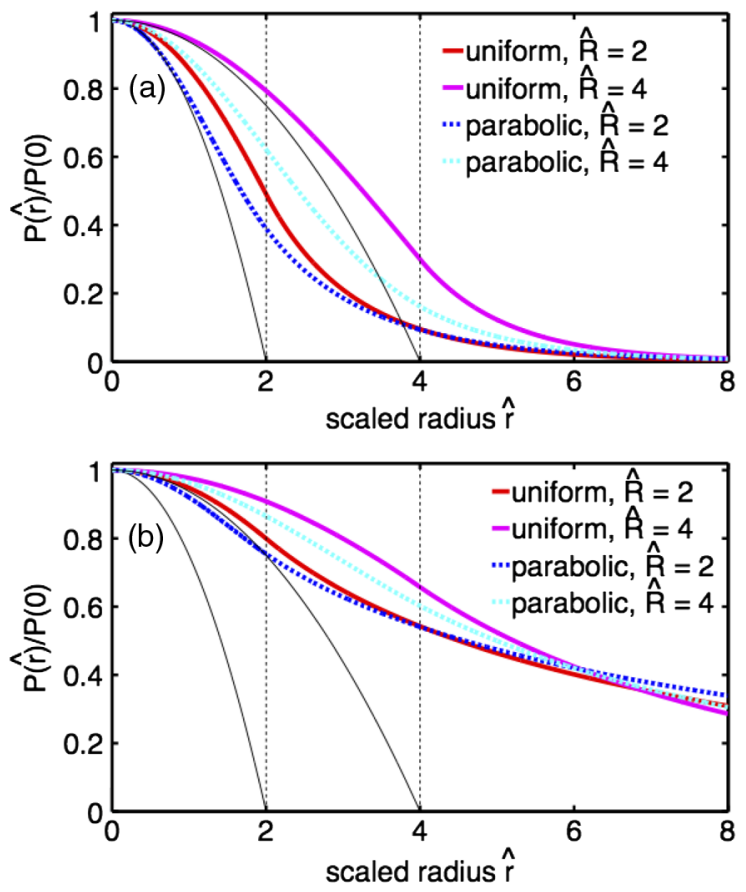

FIG. 9. The transverse radiation field profiles (colored) for two different electron beam sizes $(\hat{R}=2$ and 4$)$ for the uniform and bounded parabolic $\left(k_{1}=1\right)$ electron profiles in the untapered (a) and $8 \%$ tapered (b) cases. The two thin black lines in (a) and (b) indicate the parabolic electron beam profile.

numerical effects occurred along the boundaries. In the numerical simulation the undulator tapering starts at the very beginning with an overall taper ratio of continuous and quadratic $8 \%$. Figure 10(a) shows the mountain-range plots for the evolution of the radiation intensity profile for both untapered and $8 \%$ tapered cases, where the clear broadening of the radiation field intensity in the $8 \%$ tapered case is indicated. More quantitatively Fig. 10(b) shows the evolution of the half-width half maximum of the radiation intensity profile. We see the transverse pulse broadening proceeds after $z>20 \mathrm{~m}$. Besides, more and more of the enhanced radiation field intensity due to undulator tapering is contributed from outside of the transverse electron beam than from inside of it. By quantifying the ratio of the sum of field intensity inside the transverse electron beam size (40 $\mu \mathrm{m}$ in $r$ in the example) to that outside the beam, as shown in Fig. 10(c), we can see a decrease trend in the curve for the $8 \%$ tapered case after $z>20 \mathrm{~m}$. The on-axis field intensity will therefore grow at a relatively slower rate than the transversely integrated field intensity.

To end this section we highlight the effect of undulator tapering on broadening of the radiation field profile. In the presence of a proper undulator tapering, the more trapped electrons lead to reduction of the spread $(\alpha)$ measured in the electron longitudinal phase space, while the enhanced radiation power results in a faster synchrotron oscillation $\left(\Omega_{\text {syn }}\right)$. These two factors cause the reduction of the electron bunching in spite of slight increase of on-axis field
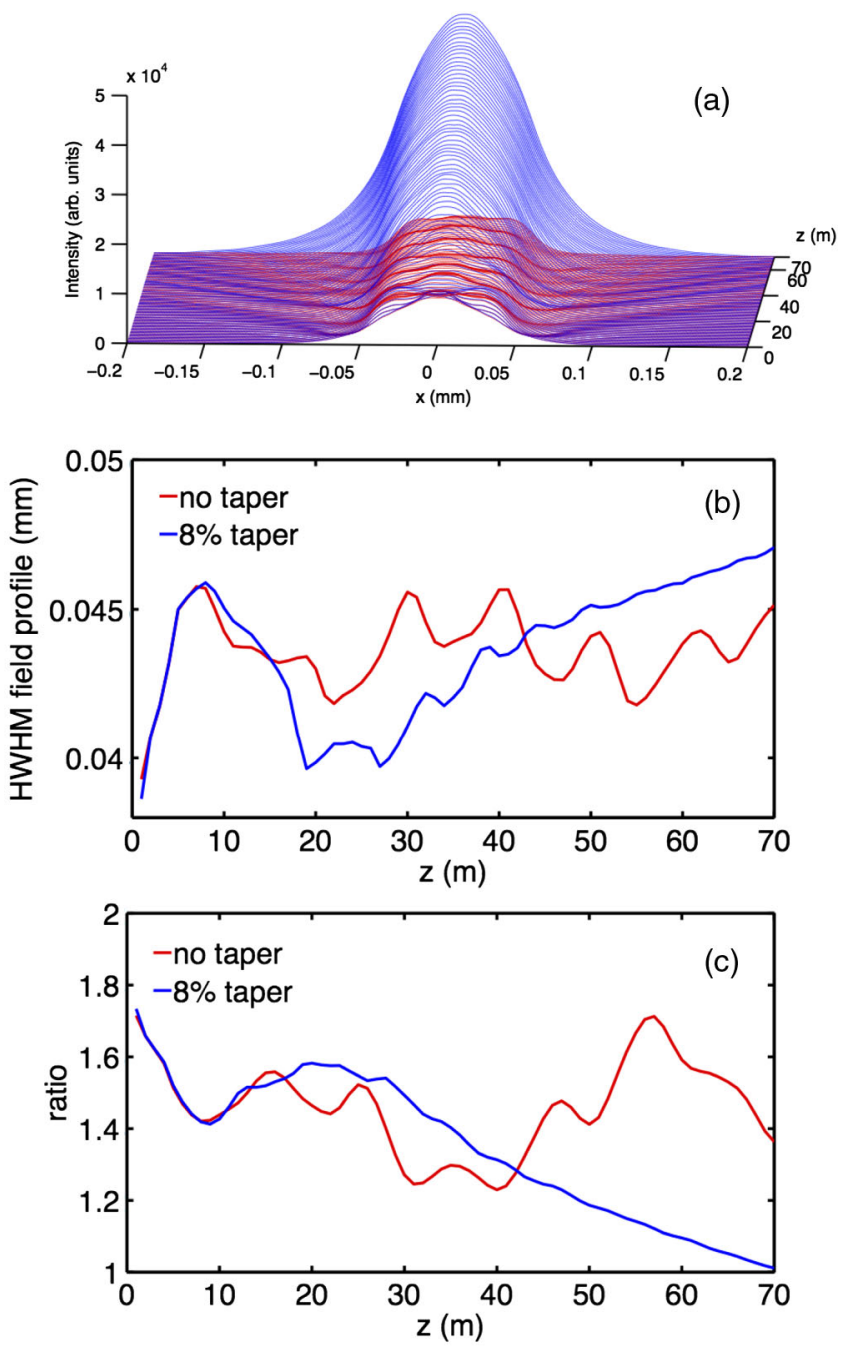

FIG. 10. Three-dimensional mountain-range plot (a) for the evolution of the radiation field intensity for the untapered (red) and $8 \%$ tapered (blue) cases. The initial transverse electron beam profile is assumed uniform within $\approx \pm 28 \mu \mathrm{m}$ in $x$ and $y$ (or $\approx \pm 40 \mu \mathrm{m}$ in $r$ ). (b) The $z$-dependence of the half-width half maximum of the intensity profile. (c) The $z$-dependence of the ratio of the integrated field intensity outside the electron beam to that inside the beam.

[Eq. (24)], weaken the effective transverse guiding [ $\mu$ in Eq. (37) or $\delta$ in (46)], and eventually lead to the broadening of the radiation field.

\section{SUMMARY AND DISCUSSION}

In this paper we have analyzed the postsaturation dynamics in a single-pass high-gain tapered FEL, including the power efficiency, the induced energy spread, and the radiation field intensity as a function of the scaled transverse electron beam size and the level of undulator tapering. By taking advantage of two integrals of the motion, one from the energy conservation and the other from the action variable based on the adiabatic invariance of the undulator 
tapering, we have studied two cases that allow analytical solutions of the radiation field: the uniform and the bounded parabolic transverse electron beam profiles. The self-consistent solutions allow us to clearly see the dependences of the FEL power efficiency, the induced energy spread increase, and the broadening of the radiation field intensity on both the transverse electron beam size and the taper ratio.

It is found that the tapered power efficiency has weak dependence on the transverse electron beam size and can be greatly improved by virtue of undulator tapering up to its total taper ratio prior to occurrence of significant electron detrapping. In the presence of undulator tapering, besides the total power is enhanced (compared with the untapered case), it is also found that more and more the field intensity is contributed from outside of the transverse electron beam than from inside of it, consistent with the numerical observation in Ref. [53]. Finally we use a full three-dimensional timeindependent simulation [52] to illustrate the spatial evolution of the radiation field intensity for an untapered and a $8 \%$ tapered case. The results are consistent with the conclusion made in our theoretical analysis for broadening of the radiation beam profile in the presence of undulator tapering.

As a note for discussion, although we have extended the previous work $[42,43]$ to study of tapered FEL dynamics, it is important to take the effect of electron detrapping into consideration. For example, when in the regime of deep undulator tapering or of large taper ratio, the electron detrapping will become significant. To our knowledge, an analytical estimation of the trapping/detrapping efficiency as a function of the introduced dependences (e.g., taper ratio, transverse beam size and so on) in similar analyses is not yet available in literature. Furthermore, in spite of twodimensional Laplacian operator in the wave equation to account for diffraction effect, the electron dynamics is not self-consistently included in two (or three) dimensional treatment. Although the finite transverse electron beam size is taken into account, the angular divergence (or the finite beam emittance) is neglected. To include the transverse electron dynamics, the betatron oscillation due to external focusing elements should also be incorporated. Theoretical studies in a more complete postsaturation tapered FEL system with inclusion of electron transverse dynamics is in our plan.

As a final comment, there has recently been an interest in shaping the electron transverse distribution utilizing advanced laser techniques (see for example Ref. [54] and references therein). The analysis here may provide further insights for its application to maximize the tapered FEL performance. As for an arbitrary transverse electron beam profile, solving the corresponding 2-D field equation [Eq. (27)] may be possible by means of the so-called multilayer approximation method [9]. Such a semianalytical approach can be much more efficient than direct, full three-dimensional numerical simulations.

\section{ACKNOWLEDGMENTS}

The work was supported by the US Department of Energy (DOE) under Contract No. DE-AC02-76SF00515 and the US DOE Office of Science Early Career Research Program Grant No. FWP-2013-SLAC-100164.

[1] H. N. Chapman et al., Femtosecond x-ray protein nanocrystallography, Nature (London) 470, 73 (2011).

[2] M. Marvin Seibert et al., Single mimivirus particles intercepted and imaged with an x-ray laser, Nature (London) 470, 78 (2011).

[3] A. Fratalocchi and G. Ruocco, Single-Molecule Imaging with X-ray Free-Electron Lasers: Dream or Reality?, Phys. Rev. Lett. 106, 105504 (2011).

[4] P. Emma et al., First lasing and operation of an ångstromwavelength free-electron laser, Nat. Photonics 4, 641 (2010).

[5] Tetsuya Ishikawa et al., A compact x-ray free-electron laser emitting in the sub-ångström region, Nat. Photonics 6, 540 (2012).

[6] E. Allaria et al., Two-stage seeded soft-x-ray free-electron laser, Nat. Photonics 7, 913 (2013).

[7] T. Hara, Fully coherent soft x-rays at fermi, Nat. Photonics 7, 852 (2013).

[8] K.-J. Kim, Three-Dimensional Analysis of Coherent Amplification and Self-Amplified Spontaneous Emission in Free-Electron Lasers, Phys. Rev. Lett. 57, 1871 (1986).

[9] E. L. Saldin, E. V. Schneidmiller, and M. V. Yurkov, The Physics of Free Electron Lasers (Springer-Verlag, Berlin Heidelberg, 2000).

[10] P. Schmuser, M. Dohlus, J. Rossbach, and C. Behrens, Free-Electron Lasers in the Ultraviolet and X-ray Regime: Physical Principles, Experimental Results, Technical Realization, Springer Tracts in Modern Physics (Springer, New York, 2014).

[11] K.-J. Kim, Z. Huang, and R. Lindberg, Synchrotron Radiation and Free-Electron Lasers: Principles of Coherent X-ray Generation (Cambridge University Press, Cambridge, England, 2017).

[12] L. H. Yu, Generation of intense uv radiation by subharmonically seeded single-pass free-electron lasers, Phys. Rev. A 44, 5178 (1991).

[13] L.-H. Yu et al., High-gain harmonic-generation freeelectron laser, Science 289, 932 (2000).

[14] G. Stupakov, Using the Beam-Echo Effect for Generation of Short-Wavelength Radiation, Phys. Rev. Lett. 102, 074801 (2009).

[15] D. Xiang and G. Stupakov, Echo-enabled harmonic generation free electron laser, Phys. Rev. ST Accel. Beams 12, 030702 (2009).

[16] J. Feldhaus, E. L. Saldin, J. R. Schneider, E. A. Schneidmiller, and M. V. Yurkov, Possible application of $\mathrm{x}$-ray optical elements for reducing the spectral bandwidth of an x-ray sase fel, Opt. Commun. 140, 341 (1997). 
[17] G. Geloni, V. Kocharyan, and E. Saldin, A novel selfseeding scheme for hard x-ray fels, J. Mod. Opt. 58, 1391 (2011).

[18] J. Amann et al., Demonstration of self-seeding in a hard-Xray free-electron laser, Nat. Photonics 6, 693 (2012).

[19] W. M. Fawley, J. Frisch, Z. Huang, Y. Jiao, H.-D. Nuhn, C. Pellegrini, S. Reiche, and J. Wu, Toward TW-level, Hard X-ray pulses at LCLS, in Proceedings of FEL2011, Shanghai, China, 2011 (JACoW, Shanghai, 2011), p. 160, http://www.jacow.org.

[20] N. Kroll, P. Morton, and M. Rosenbluth, Free-electron lasers with variable parameter wigglers, IEEE J. Quantum Electron. 17, 1436 (1981).

[21] D. Ratner, A. Chao, and Z. Huang, Enhancing FEL power with phase shifters, in Proceedings of FEL2007, Novosibirsk, Russia, 2007 (JACoW, Novosibirsk, 2007), p. 69, http://www.jacow.org.

[22] A. Mak, F. Curbis, and S. Werin, Phase jump method for efficiency enhancement in free-electron lasers, Phys. Rev. Accel. Beams 20, 060703 (2017).

[23] Y. Jiao, J. Wu, Y. Cai, A. W. Chao, W. M. Fawley, J. Frisch, Z. Huang, H.-D. Nuhn, C. Pellegrini, and S. Reiche, Modeling and multidimensional optimization of a tapered free electron laser, Phys. Rev. ST Accel. Beams 15, 050704 (2012).

[24] C. Emma, K. Fang, J. Wu, and C. Pellegrini, High efficiency, multiterawatt x-ray free electron lasers, Phys. Rev. Accel. Beams 19, 020705 (2016).

[25] J. Wu, N. Hu, H. Setiawan, X. Huang, T. O. Raubenheimer, Y. Jiao, G. Yu, A. Mandlekar, S. Spampinati, K. Fang, C. Chu, and J. Qiang, Multi-dimensional optimization of a terawatt seeded tapered free electron laser with a multiobjective genetic algorithm, Nucl. Instrum. Methods Phys. Res., Sect. A 846, 56 (2017).

[26] G. T. Moore, The high-gain regime of the free electron laser, Nucl. Instrum. Methods Phys. Res., Sect. A 239, 19 (1985).

[27] E. T. Scharlemann, A. M. Sessler, and J. S. Wurtele, Optical Guiding in a Free-Electron Laser, Phys. Rev. Lett. 54, 1925 (1985).

[28] S. Krinsky and L. H. Yu, Output power in guided modes for amplified spontaneous emission in a single-pass freeelectron laser, Phys. Rev. A 35, 3406 (1987).

[29] P. Sprangle, A. Ting, and C. M. Tang, Radiation Focusing and Guiding with Application to the Free Electron Laser, Phys. Rev. Lett. 59, 202 (1987).

[30] A. Bhattacharjee, S. Y. Cai, S. P. Chang, J. W. Dodd, and T. C. Marshallm, Observations of Optical Guiding in a Raman Free-Electron Laser, Phys. Rev. Lett. 60, 1254 (1988).

[31] L. H. Yu, S. Krinsky, and R. L. Gluckstern, Calculation of Universal Scaling Function for Free-Electron-Laser Gain, Phys. Rev. Lett. 64, 3011 (1990).

[32] Z. Huang and K.-J. Kim, Solution to the initial value problem for a high-gain fel via van kampen's method, Nucl. Instrum. Methods Phys. Res., Sect. A 475, 59 (2001).

[33] J. Wu and L. H. Yu, Eigenmodes and mode competition in a high-gain free-electron laser including alternating-gradient focusing, Nucl. Instrum. Methods Phys. Res., Sect. A 475, 79 (2001).
[34] R. Bonifacio, C. Pellegrini, and L. M. Narducci, Collective instabilities and high-gain regime in a free electron laser, Opt. Commun. 50, 373 (1984).

[35] R. C. Davidson and J. S. Wurtele, Single-particle analysis of the free-electron laser sideband instability for primary electromagnetic wave with constant phase and slowly varying phase, Phys. Fluids 30, 557 (1987).

[36] W. M. Sharp and S. S. Yu, Two-dimensional vlasov treatment of free-electron laser sidebands, Phys. Fluids B 2, 581 (1990).

[37] R. P. Pilla and A. Bhattacharjee, Elimination of the sideband instability in variable-parameter free-electron lasers and inverse free-electron lasers, Phys. Plasmas 1, 390 (1994).

[38] S. Riyopoulos, Sideband suppression in tapered wiggler free electron lasers including thermal spreads, Phys. Plasmas 7, 1586 (2000).

[39] S. Isermann and R. Graham, Suppression of the sideband instability in tapered free-electron lasers, Phys. Rev. A 45, 4050 (1992).

[40] C.-Y. Tsai, J. Wu, C. Yang, M. Yoon, and G. Zhou, Sideband instability analysis based on a one-dimensional high-gain free electron laser model, Phys. Rev. Accel. Beams 20, 120702 (2017).

[41] E. A. Schneidmiller and M. V. Yurkov, Optimization of a high efficiency free electron laser amplifier, Phys. Rev. ST Accel. Beams 18, 030705 (2015).

[42] R. L. Gluckstern, S. Krinsky, and H. Okamoto, Analysis of the saturation of a high-gain free-electron laser, Phys. Rev. E 47, 4412 (1993).

[43] Z. Huang and K.-J. Kim, Transverse and temporal characteristics of a high-gain free-electron laser in the saturation regime, Nucl. Instrum. Methods Phys. Res., Sect. A 483, 504 (2002).

[44] T. M. Antonsen and B. Levush, Optical guiding in the separable beam limit, Nucl. Instrum. Methods Phys. Res., Sect. A 272, 472 (1988).

[45] N. Metzler, T. Antonsen Jr., and B. Levush, Nonlinear optical guiding in the separable beam limit, Phys. Fluids B: Plasma Phys. 2, 1038 (1990).

[46] B. W. J. McNeil, G. R. M. Robb, M. W. Poole, and N. R. Thompson, Harmonic Lasing in a Free-Electron-Laser Amplifier, Phys. Rev. Lett. 96, 084801 (2006).

[47] E. A. Schneidmiller and M. V. Yurkov, Harmonic lasing in $\mathrm{X}$-ray free electron lasers, Phys. Rev. ST Accel. Beams 15, 080702 (2012).

[48] E. A. Schneidmiller, B. Faatz, M. Kuhlmann, J. RönschSchulenburg, S. Schreiber, M. Tischer, and M. V. Yurkov, First operation of a harmonic lasing self-seeded free electron laser, Phys. Rev. Accel. Beams 20, 020705 (2017).

[49] D. R. Nicholson, Introduction to Plasma Theory (Wiley, New York, 1983).

[50] B. E. A. Saleh and M. C. Teich, Fundamentals of Photonics, Wiley Series in Pure and Applied Optics (Wiley, New York, 1991).

[51] J. Mathews and R. L. Walker, Mathematical Methods of Physics, 2nd ed. (W.A. Benjamin, New York, 1970). 
[52] S. Reiche, Genesis 1.3: A fully 3d time-dependent fel simulation code, Nucl. Instrum. Methods Phys. Res., Sect. A 429, 243 (1999).

[53] B. Hafizi, A. Ting, P. Sprangle, and C. M. Tang, Efficiency Enhancement and Optical Guiding in a Tapered High-Power Finite-Pulse Free-Electron Laser, Phys. Rev. Lett. 64, 180 (1990).
[54] S. Li, S. Alverson, D. Bohler, A. Egger, A. Fry, S. Gilevich, Z. Huang, A. Miahnahri, D. Ratner, J. Robinson, and F. Zhou, Ultraviolet laser transverse profile shaping for improving X-ray free electron laser performance, Phys. Rev. Accel. Beams 20, 080704 (2017). 